Review began 01/20/2022 Review ended 01/25/2022 Published 01/27/2022

\section{() Copyright 2022}

Otaka et al. This is an open access article distributed under the terms of the Creative Commons Attribution License CC-BY 4.0. which permits unrestricted use, distribution, and reproduction in any medium, provided the original author and source are credited.

\title{
Myeloperoxidase Antineutrophil Cytoplasmic Antibody-Associated Renal-Limited Vasculitis in a Young Adult Woman
}

\author{
Yukihiro Otaka ${ }^{1,2}$, Daiki Uchida ${ }^{1}$, Kinue Shimizu-Arii ${ }^{3}$, Nobuyoshi Ishiyama ${ }^{1}$, Keiko Kawai-Kowase ${ }^{2}$ \\ 1. General Internal Medicine, Hidaka-kai Hidaka Hospital, Gunma, JPN 2. General Medicine, Gunma University \\ Graduate School of Medicine, Gunma, JPN 3. Pathology, Hidaka-kai Hidaka Hospital, Gunma, JPN
}

Corresponding author: Yukihiro Otaka, m13702059@gunma-u.ac.jp

\begin{abstract}
Antineutrophil cytoplasmic antibody (ANCA)-associated renal-limited vasculitis (RLV) is a minor subtype of small vessel vasculitis characterized by the inflammation of blood vessels, tissue damage, and loss of renal function localized in the kidney without systemic involvements. Here, we report a case of myeloperoxidase (MPO) ANCA-associated RLV in a young adult woman in Japan presenting chronic hematuria and newly overt proteinuria. Percutaneous renal biopsy revealed focal fibro-cellular crescent glomerulonephritis and the absence of other small vasculitides, tubular atrophy, and interstitial fibrosis. Therapeutic intravenous methylprednisolone pulse followed by oral prednisolone was administered as a remission induction. The patient's serum MPO-ANCA level gradually decreased, coinciding with dramatic changes in proteinuria and hematuria after therapeutic glucocorticoid administration. Renal function was maintained within the normal range, and disease activity was well-tolerated throughout the follow-up period for more than 14 weeks. While the incidence of RLV is rare among younger patients, it occurs with asymptomatic hematuria and proteinuria, which is important in differentiating RLV from typical glomerulonephritis. The overall prognosis of ANCA-associated RLV potentially depends on the severity of extrarenal involvements. Early diagnosis, appropriate treatment, and regular maintenance are essential for controlling and treating RLV. Due to the nontypical case presented here, further investigation is recommended to improve the diagnosis strategies and treatment options for this disease.
\end{abstract}

Categories: Internal Medicine, Pathology, Nephrology

Keywords: renal-limited vasculitis, myeloperoxidase, microscopic polyangiitis, focal category, fibro-cellular crescent glomerulonephritis, antineutrophil cytoplasmic antibody

\section{Introduction}

Antineutrophil cytoplasmic antibody (ANCA)-associated vasculitis (AAV) is a rare disease characterized by the inflammation of blood vessels, endothelial injury, and damage of surrounding tissues [1]. Along with small vessel vasculitis, microscopic polyangiitis (MPA) features a loss of tolerance to neutrophil primary granule proteins, mainly myeloperoxidase (MPO). Instances of MPA typically occur in elderly populations and are accompanied by rapidly progressing glomerulonephritis (GN) with hematuria, proteinuria, and a progressive loss of renal function over a short period [2].

ANCA-associated renal-limited vasculitis (RLV) is a small vessel vasculitis localized in the kidney without systemic involvement [1]. ANCA-associated RLV shows many features that suggest it represents a renallimited form of MPA. Additionally, ANCA-associated RLV showed relatively better outcomes compared with typical MPA or granulomatosis presenting with polyangiitis in terms of renal relapse, long-term dialysis, kidney transplantation, and mortality [3]. The mean age of RLV diagnosis was 60 years, with younger cases being uncommon [3-4]. Several studies of juvenile or young adult patients with AAV/ANCA-associated RLV have been reported [5]; however, more research is required to further characterize the long-term outcome of the disease.

\section{Case Presentation}

A 23-year-old Japanese woman with a three-year history of asymptomatic microscopic hematuria was referred to our department due to precipitating overt proteinuria and loss of appetite. The patient had a history of intermittent microscopic hematuria in her late childhood but was not referred to a doctor for further examination until recently. The patient's microscopic hematuria had been documented at an annual medical checkup three years before admission, and she was referred to a urologist for further investigation. The patient displayed iron-deficiency anemia and chronic pruritus as comorbidities for the preceding six months, which had been treated with oral iron supplement and antihistamines. The patient was a nonsmoker and an occasional light drinker.

Upon admission, the patient's body temperature was $37.3^{\circ} \mathrm{C}$, blood pressure was $150 / 98 \mathrm{mmHg}$, and pulse rate was 103 beats per minute. Height was measured at $163.0 \mathrm{~cm}$, and body weight was $52.8 \mathrm{~kg}$ (body-mass 


\section{Cureus}

index was 19.9). The patient had lost $3.6 \mathrm{~kg}$ during the preceding four months due to a prolonged loss of appetite. Other vital signs and physical findings were unremarkable, presenting no skin lesions or neurological disorders. Laboratory tests revealed a slight increase in white blood cell count but no evidence of systemic inflammation was seen (Table 1). 


\section{Cureus}

\begin{tabular}{|c|c|c|}
\hline Items & Values & \\
\hline WBC count & 11,170 & $/ \mu \mathrm{L}$ \\
\hline RBC count & $4.64 \times 10^{6}$ & $/ \mu \mathrm{L}$ \\
\hline Reticulocyte & 143,376 & $/ \mu \mathrm{L}$ \\
\hline Hemoglobin & 12.3 & $\mathrm{~g} / \mathrm{dL}$ \\
\hline Hematocrit & 39.2 & $\%$ \\
\hline Platelet count & 534,000 & $/ \mu \mathrm{L}$ \\
\hline Total protein & 7.2 & $\mathrm{~g} / \mathrm{dL}$ \\
\hline Albumin & 4.4 & $\mathrm{~g} / \mathrm{dL}$ \\
\hline Lactate dehydrogenase & 146 & IU/L \\
\hline Urea nitrogen & 10.9 & $\mathrm{mg} / \mathrm{dL}$ \\
\hline Creatinine & 0.57 & $\mathrm{mg} / \mathrm{dL}$ \\
\hline eGFR creatinine & 108 & $\mathrm{~mL} / \mathrm{min} / 1.73 \mathrm{~m}^{2}$ \\
\hline C-reactive protein & $<0.05$ & $\mathrm{mg} / \mathrm{dL}$ \\
\hline Ferritin & 106.3 & $\mathrm{ng} / \mathrm{mL}$ \\
\hline Rheumatoid factor (LA) & 3 & IU/mL \\
\hline $\lg A$ & 127 & $\mathrm{mg} / \mathrm{dL}$ \\
\hline $\operatorname{lgE}$ & 113 & IU/mL \\
\hline $\lg G$ & 1,325 & $\mathrm{mg} / \mathrm{dL}$ \\
\hline $\operatorname{lgM}$ & 133 & $\mathrm{mg} / \mathrm{dL}$ \\
\hline CH50 & 57 & $\mathrm{U} / \mathrm{mL}$ \\
\hline Complement component 3 & 99 & $\mathrm{mg} / \mathrm{dL}$ \\
\hline Complement component 4 & 15.0 & $\mathrm{mg} / \mathrm{dL}$ \\
\hline Anti-nuclear antibody & Less than 1:40 & \\
\hline MPO-ANCA (FEIA) & 681.0 & IU/mL \\
\hline PR3-ANCA (FEIA) & $<0.5$ & IU/mL \\
\hline Anti-GBM antibody (FEIA) & 0.6 & $\mathrm{U} / \mathrm{mL}$ \\
\hline Cryoglobulin & Negative & \\
\hline Anti-dsDNA antibody (FEIA) & 1.1 & IU/mL \\
\hline Hepatitis-B surface antigen & Negative & \\
\hline Hepatitis-C virus antibody & Negative & \\
\hline
\end{tabular}

\section{TABLE 1: Laboratory findings on admission}

Abbreviations: ANCA, anti-neutrophil cytoplasmic antibody; $\mathrm{CH} 50,50 \%$ hemolytic complement activity; dsDNA, double-stranded deoxyribonucleic acid; eGFR, estimated glomerular filtration rate calculated by the Japanese equation for Modification of Diet in Renal Disease [6]); FEIA, fluorescence enzyme immunoassay; GBM, glomerular basement membrane; Ig, immunoglobulin; LA, latex agglutination turbidimetric immunoassay; MPO, myeloperoxidase; PR3, proteinase 3; RBC, red blood cell; WBC, white blood cell

Progressive proteinuria ( $908.8 \mathrm{mg}$ per day) and microscopic hematuria (50-99 of red blood cells per high power field) were observed; however, subsequent deformation of urinary red blood cells and casts were not detected. Renal function was preserved (24-hour creatinine clearance, $160 \mathrm{~mL} / \mathrm{min}$ ), and the iron-deficiency anemia was well-controlled with medication. Serum MPO-ANCA presented a high titer of $681.0 \mathrm{IU} / \mathrm{mL}$ well 


\section{Cureus}

above the normal range $(<3.5 \mathrm{IU} / \mathrm{mL})$. There was no evidence of lung consolidation, interstitial changes, or honeycomb signs on her chest X-ray and computed tomography.

Percutaneous renal biopsy specimens contained 40 glomeruli, including three adhesive lesions, three cases of segmental sclerosis, and one fibrocellular crescent (Figure 1a).

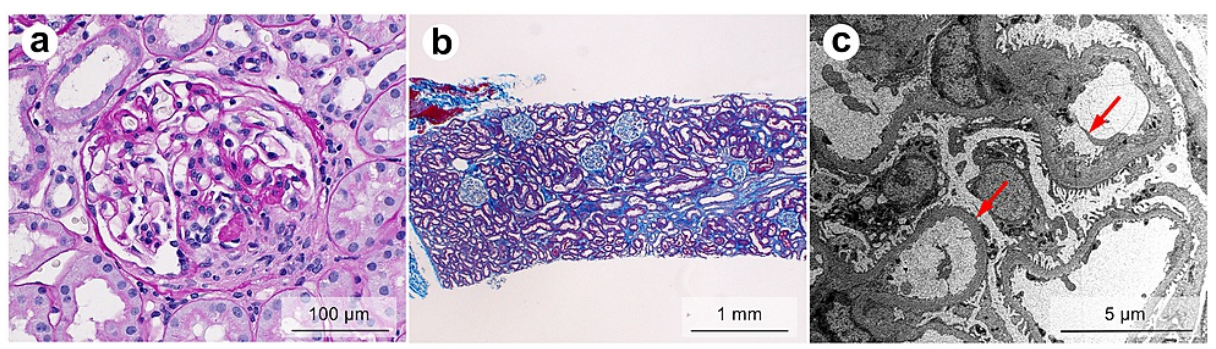

\section{FIGURE 1: Histopathology of renal biopsy specimens}

(a) Focal fibro-cellular crescent formation (periodic acid-Schiff stain); (b) Unremarkable tubular atrophy and interstitial fibrosis (Masson's trichrome stain); (c) Focal widening of the subendothelial spaces in the glomerulocapillary (arrows), lamination, and uneven thickening and thinning of basement membrane (electron microscopy)

The rupture of the glomerular capillary wall, fibrin precipitation, and fibrin thrombus formation was observed in some glomeruli, showing focal necrotizing GN. There were no inflammatory changes, no double contour or spike formation of the basement membrane, and no enlargement of the mesangial region. Intratubular erythrocyte casts were observed, but there was no atrophy of renal tubules, and interstitial inflammation or fibrosis was unremarkable (Figure $1 b$ ). No evidence of vascular sclerosis or vasculitis was found in the interlobular arteries, venules, or arterioles. A small deposit of immunoglobulin $\mathrm{M}$ in the mesangial region was observed, but otherwise, few deposits were seen via immunofluorescent staining. Electron microscopy did reveal focal glomerular collapse, widening on the subendothelial spaces, lamination, and thickening and wrinkling of the basement membrane, representing the effects of vasculitis (Figure 1c). Electron dense deposits were not observed.

Based on the patient's clinical and pathological findings, the patient was diagnosed as MPO-ANCAassociated RLV, with a high titer of circulating MPO-ANCA and pauci-immune necrotizing GN. The Initial Birmingham Vasculitis Activity Score-2003 (BVAS-2003) at diagnosis was 11, accorded by newly diagnosed hypertension (+4), persistent weight loss (+2), proteinuria (+2), and hematuria (+3). The remission induction therapy comprised intravenous methylprednisolone pulse ( $1 \mathrm{~g}$ per day) followed by oral prednisolone (50 $\mathrm{mg}$ daily for 2 weeks, then tapered gradually). Although proteinuria and hematuria were dramatically reduced after glucocorticoid administration (Figures $2 a-2 b$ ), serum MPO-ANCA remained well above the normal limit; however, the titer progressively decreased along with maintenance therapy (Figure 2c).

a

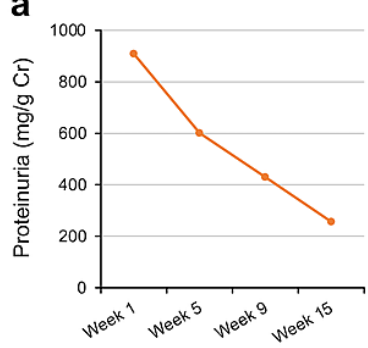

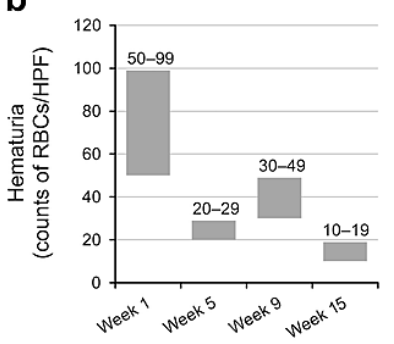

C

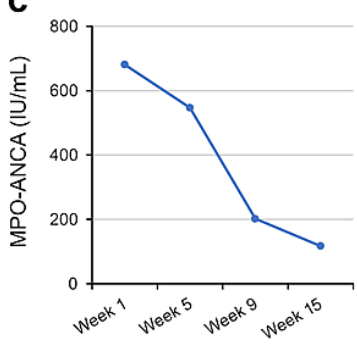

FIGURE 2: The changes in proteinuria, microscopic hematuria, and serum myeloperoxidase-antineutrophil cytoplasmic antibody (MPOANCA) throughout treatments

During the follow-up period over the next 14 weeks, the estimated glomerular filtration rate was maintained at the normal range between 90 and $110 \mathrm{~mL} / \mathrm{min} / 1.73 \mathrm{~m}^{2}$, and the reassessed BVAS-2003 was 5 , accorded by the still persistent proteinuria and microscopic hematuria at week 15 . 


\section{Discussion}

$\mathrm{AAV}$ is a rare disease characterized by microvascular endothelial inflammation leading to extravascular inflammation, progressive injury, tissue damage, fibrosis, and loss of function. Among the small vessel vasculitis defined by the 2012 revised Chapel Hill Consensus Conference [1], MPA features a loss of tolerance to neutrophil primary granule proteins, which predominate MPO in $60 \%$ of cases. RLV, a minor subtype of single-organ AAVs, is characterized by small vessel vasculitis localized in the kidney occurring in the absence of systemic involvement [1]. Approximately $80 \%$ of patients with RLV will be positive for ANCA, predominantly MPO-ANCA, which has many features to suggest that it represents a renal-limited form of MPA [3]. The incidence of MPA was 0.5-24.0 cases per one million person-years, and the typical age of disease onset is reported at 55-75 years [2]. Similarly, the mean age at diagnosis of RLV was 59.8 years [3-4]. The sex ratio is estimated to be roughly $1: 1$, and geographically, MPA predominates in Asian regions such as Japan and China.

In a single-center study for rapidly progressive GN, pauci-immune crescentic GN, including MPA and RLV, was observed in $42 \%$ of younger patients aged between one and 20 years [7]. Moreover, unlike in adults, AAV in children is likely to be more predominant among women rather than men [8-9]. This case showed a previously healthy young adult woman with a final diagnosis of MPO-ANCA-associated RLV. Although the incidence of RLV at a young age is extremely rare, it may occur with asymptomatic hematuria and overt proteinuria, the comorbidity of which is important as one of the differentials from other typical GNs such as immunoglobulin A nephropathy.

Among the four patterns of glomerular lesions defined by a histopathological classification in AAV, the focal category ( $\geqslant 50 \%$ normal glomeruli regardless of tubulointerstitial lesions or renal function), which was seen in this case, showed the best prognosis, i.e., relatively preserved renal function and favorable renal outcome, compared with the other types of injuries [10]. More recent studies have shown that a renal risk score using the percentages of normal glomeruli, tubular atrophy/interstitial fibrosis, and estimated glomerular filtration rate at the time of diagnosis will predict renal survival and the risk of end-stage renal disease (ESRD) [11]. Among these parameters, the percentage of normal glomeruli was the strongest independent predictor of mortality from ESRD [11].

\section{Conclusions}

In summary, the prognosis of MPA or ANCA-associated RLV potentially depends on the severity of organ involvement. Early diagnosis, appropriate treatment, and constant maintenance are essential for all patients, including adolescents and children. Further investigation is required to improve the strategies of diagnosis and treatment options for this disease, as its occurrence in younger patients is poorly understood.

\section{Additional Information \\ Disclosures}

Human subjects: Consent was obtained or waived by all participants in this study. The ethical review board of Hidaka Hospital issued approval 344. All procedures performed in the study involving human participants were in accordance with the ethical standards of the institutional research committee at which the study was conducted and with the 1964 Helsinki declaration and its later amendments or comparable ethical standards. All presented materials have been fully anonymized to protect the participant's identity. Conflicts of interest: In compliance with the ICMJE uniform disclosure form, all authors declare the following: Payment/services info: All authors have declared that no financial support was received from any organization for the submitted work. Financial relationships: All authors have declared that they have no financial relationships at present or within the previous three years with any organizations that might have an interest in the submitted work. Other relationships: All authors have declared that there are no other relationships or activities that could appear to have influenced the submitted work.

\section{Acknowledgements}

The authors thank Dr. Ayaka Hirota, Dr. Shoma Yamauchi, Dr. Yuki Kuribara, and Dr. Kota Otachi (General Internal Medicine, Hidaka-kai Hidaka Hospital) for their aid in patient management, and Dr. Takaaki Tsutsui and Dr. Nobuo Nagano (Kidney Disease and Dialysis Center, Hidaka-kai Hidaka Hospital) and Dr. Yuko Iwabuchi-Nakaya (Department of Nephrology, Tokyo Women's Medical University, Tokyo, Japan) for critical review of the manuscript. We also thank Enago (www.enago.jp) for its English language review.

\section{References}

1. Jennette JC, Falk RJ, Bacon PA, et al.: 2012 revised International Chapel Hill Consensus Conference Nomenclature of Vasculitides. Arthritis Rheum. 2013, 65:1-11. 10.1002/art.37715

2. Kitching AR, Anders HJ, Basu N, et al.: ANCA-associated vasculitis. Nat Rev Dis Primers. 2020, 6:71. 10.1038/s41572-020-0204-y

3. Jennette JC, Nachman PH: ANCA glomerulonephritis and vasculitis. Clin J Am Soc Nephrol. 2017, 12:168091. 10.2215/CJN.02500317

4. Lionaki S, Blyth ER, Hogan SL, et al.: Classification of antineutrophil cytoplasmic autoantibody vasculitides: 


\section{Cureus}

the role of antineutrophil cytoplasmic autoantibody specificity for myeloperoxidase or proteinase 3 in disease recognition and prognosis. Arthritis Rheum. 2012, 64:3452-62. 10.1002/art.34562

5. Yamauchi H, Shiotsu Y, Nakamura I, et al.: MPO-ANCA-associated glomerulonephritis diagnosed by medical examination in a young adult: a case report [Article in Japanese]. Jpn J Nephrol. 2019, 61:1115-20.

6. Matsuo S, Imai E, Horio M, et al.: Revised equations for estimated GFR from serum creatinine in Japan . Am J Kidney Dis. 2009, 53:982-92. 10.1053/j.ajkd.2008.12.034

7. Jennette JC: Rapidly progressive crescentic glomerulonephritis. Kidney Int. 2003, 63:1164-77. 10.1046/.1523-1755.2003.00843.x

8. Hattori M, Kurayama H, Koitabashi Y: Antineutrophil cytoplasmic autoantibody-associated glomerulonephritis in children. J Am Soc Nephrol. 2001, 12:1493-500. 10.1681/ASN.V1271493

9. Plumb LA, Oni L, Marks SD, Tullus K: Paediatric anti-neutrophil cytoplasmic antibody (ANCA)-associated vasculitis: an update on renal management. Pediatr Nephrol. 2018, 33:25-39. 10.1007/s00467-016-3559-2

10. Berden AE, Ferrario F, Hagen EC, et al.: Histopathologic classification of ANCA-associated glomerulonephritis. J Am Soc Nephrol. 2010, 21:1628-36. 10.1681/ASN.2010050477

11. Brix SR, Noriega M, Tennstedt P, et al.: Development and validation of a renal risk score in ANCAassociated glomerulonephritis. Kidney Int. 2018, 94:1177-88. 10.1016/j.kint.2018.07.020 